\title{
Spitzer IRAC Colors of Nebulae Associated with Star-Forming Regions
}

\author{
Yoichi Itoh1, Yumiko Oasa ${ }^{2}$ \\ ${ }^{1}$ Nishi-Harima Astronomical Observatory, Center for Astronomy, University of Hyogo, Sayo, Hyogo, Japan \\ ${ }^{2}$ Faculty of Education, Saitama University, Saitama, Japan \\ Email: yitoh@nhao.jp
}

How to cite this paper: Itoh, Y. and Oasa, Y. (2019) Spitzer IRAC Colors of Nebulae Associated with Star-Forming Regions. International Journal of Astronomy and Astrophysics, 9, 39-50.

https://doi.org/10.4236/ijaa.2019.91004

Received: January 27, 2019

Accepted: March 4, 2019

Published: March 7, 2019

Copyright $\odot 2019$ by author(s) and Scientific Research Publishing Inc. This work is licensed under the Creative Commons Attribution International License (CC BY 4.0).

http://creativecommons.org/licenses/by/4.0/

\begin{abstract}
Star-forming regions are often associated with nebulosity. In this study, we investigated infrared diffuse emission in Spitzer IRAC images. The infrared nebula L1527 traces outflows emanating from a low-mass protostar. The nebular color is consistent with the color of a stellar photosphere with large extinction. Nebulae around the HII region W5-East are bright in the infrared. These colors are consistent with the model color of dust containing polycyclic aromatic hydrocarbon (PAH). The strength of ultraviolet irradiation of the nebulae and the small dust fraction were deduced from the infrared colors of the nebulae. We found that the edges of the nebulae are irradiated by strong ultraviolet radiation and have abundant small dust. Dust at the surface of the molecular cloud is thought to be destroyed by ultraviolet radiation from an early-type star.
\end{abstract}

\section{Keywords}

Interstellar Medium, Dust, Extinction, HII Regions

\section{Introduction}

Dust is one of the most fundamental solid materials in the universe. It is ubiquitous, but dust grains have diverse sizes. It is widely believed that small dust is abundant in the interstellar medium. Interstellar extinction increases with decreasing wavelength in the ultraviolet (UV) and optical wavelengths, with a characteristic bump at $2175 \AA$ [1] claimed that this wavelength dependence of the extinction is well reproduced by graphite dust with a power law size distribution between 0.005 and $1 \mu \mathrm{m}$ in diameter with an exponent of $\sim-3.5$. On the other hand, an overabundance of large dust has been observed in dense regions. [2] 
found that the Lupus 3 dark cloud shines in near-infrared wavelengths. They attributed the nebular emission to scattering of starlight by dust. The observed near-infrared color was reproduced by an interstellar dust model with a higher number density for a larger size regime. [3] conducted polarimetric imaging observations of a T Tauri star, UX Tau. With a spatial resolution of 0.1 " (14 AU), they detected a strongly polarized circumstellar disk surrounding UX Tau A. It extends to $120 \mathrm{AU}$ with a polarization degree ranging from $1.6 \%$ to $66 \%$. The observed azimuthal profile of the polarization degree was not consistent with dust models using Rayleigh scattering or Mie scattering approximations. A thin disk model with nonspherical dust having a diameter of $60 \mu \mathrm{m}$ reproduced the observed azimuthal profile.

Polycyclic aromatic hydrocarbon (PAH) is a major component of dust. PAH has many broad emission features in the near- and mid-infrared wavelengths. The strength of the PAH features depends on the number of molecules, degree of ionization, and strength of the UV radiation. [4] conducted Spitzer Infrared Spectrograph (IRS) observations of NGC 7023, a reflection nebula irradiated by a Herbig Be star, HD 200775. They observed a 72" $\times 54$ " region northwest of the exciting star in the slit-scan mode. This region contains a photodissociation region (PDR) and a molecular cloud. They detected PAH emission bands between 5 and $15 \mu \mathrm{m}$ and fitted them by model spectra to determine the size, charge, composition, and hydrogen adjacency of the dust. The boundary between the PDR and the molecular cloud showed a distinct discontinuity in the PAH characteristics. In the molecular cloud, small, neutral PAHs account for the emission, whereas stable, large, symmetric, and compact PAH cations are abundant in the PDR. They also found enhancement of the 6.2 and $11.0 \mu \mathrm{m}$ emission close to the exciting star, indicating $\mathrm{PAH}$ photodehydrogenation and fragmentation.

The fluxes and shapes of PAH features can be obtained using an infrared spectrograph. However, the two-dimensional distribution of PAHs cannot be obtained except by slit-scan observations or the use of a three-dimensional spectrograph. Instead, we investigate the spatial distribution of PAHs by imaging observations. [5] (hereafter DL07) synthesized the infrared emission spectra of dust heated by starlight. They considered mixtures of amorphous silicate and graphitic dust. Small carbonaceous dust has PAH-like properties. The PAH mass fraction, starlight intensity, and fraction of the dust heated by starlight were parameters in the calculation. The results indicated that small dust has high radiation efficiency at short wavelengths. They presented model emissivities for Spitzer Infrared Array Camera (IRAC) and Multiband Imaging Photometer (MIPS) photometry. [6] constructed the spectral energy distributions (SEDs) of 65 galaxies using Spitzer IRAC and MIPS photometry and James Clerk Maxwell Telescope Submillimetre Common-User Bolometer Array photometry. They fitted the dust model spectra of DL07 to the SEDs and found that small dust with $\mathrm{PAH}$ emission is abundant in galaxies with high metallicity. 
We investigate the infrared colors of nebulae irradiated by a nearby star using archival Spitzer IRAC data. By comparison with the model colors, we consider the properties of dust in molecular clouds. In this paper, the phrase "small dust" is used for dust with less than $10^{3} \mathrm{C}$ atoms, which corresponds to a diameter of $\sim 13 \AA$, as it is used in DL07.

\section{Data}

Infrared data were taken from the Spitzer Archive Center. We used the reduced data [post-basic calibrated data (PBCD)] of $3.6 \mu \mathrm{m}$ images, $4.5 \mu \mathrm{m}$ images, and $5.8 \mu \mathrm{m}$ images (PI: Fazio Giovanni). The spatial resolution of the data is about 1.8 arc seconds. We measured the average and standard deviation of the sky region or the HII region adjacent to the nebula and then subtracted the average count from the image. Next, we made three types of mask images. The first mask image was designed to reject low signal-to-noise regions. We made it from the sky-subtracted image by replacing counts more than $10 \sigma$ above the sky count with one and counts less than $10 \sigma$ above the sky count to zero. The second mask image was designed for point source rejection. Point sources were identified on the sky-subtracted image by the DAOFIND task in the IRAF software, and the flux of each source was measured by aperture photometry. A mask was created for each source, as a large aperture mask was made for a bright object. The mask regions were set to zero, and the other regions were set to unity. We also made another point source mask image using Sextractor with the SEGMENTATION option in the CHECKIMAGE_TYPE parameter. These three types of mask images were multiplied by the sky-subtracted image so that only diffuse emission appears in the final image.

\section{Results}

\subsection{L1527}

We investigate the infrared color of a nebula without PAH emission. L1527 is a reflection nebula associated with the low-mass protostar IRAS $04368+2557$ in the Taurus molecular cloud. It is seen in edge-on geometry with bipolar outflows toward the east and west. Strong UV radiation is not expected from the central star; thus, PAH is not expected to be excited. Figure 1 shows the color-color diagram of L1527. We measured the nebular flux of $5 \times 5$ pixels $(3.0$ " $\times 3.0$ ") in each band. Error bars in the figure indicate the average values of standard deviations of the $5 \times 5$ pixel regions. The error may be overestimated because uniform color is assumed in each $5 \times 5$ pixel region. In the figure, the colors of dwarfs and giants are located around $(0,0)$ [7]. The nebular colors are plotted along the interstellar extinction vector [8] with the origin at $(0,0)$ and are around the colors of blackbodies. Those colors are consistent with the colors of Class I and Class II sources [9]. The PAH model (DL07) does not reproduce the color of L1527. We conclude that the nebula L1527 is a reflection nebula and does not contain strong PAH emission features. We also claim that the color-color 


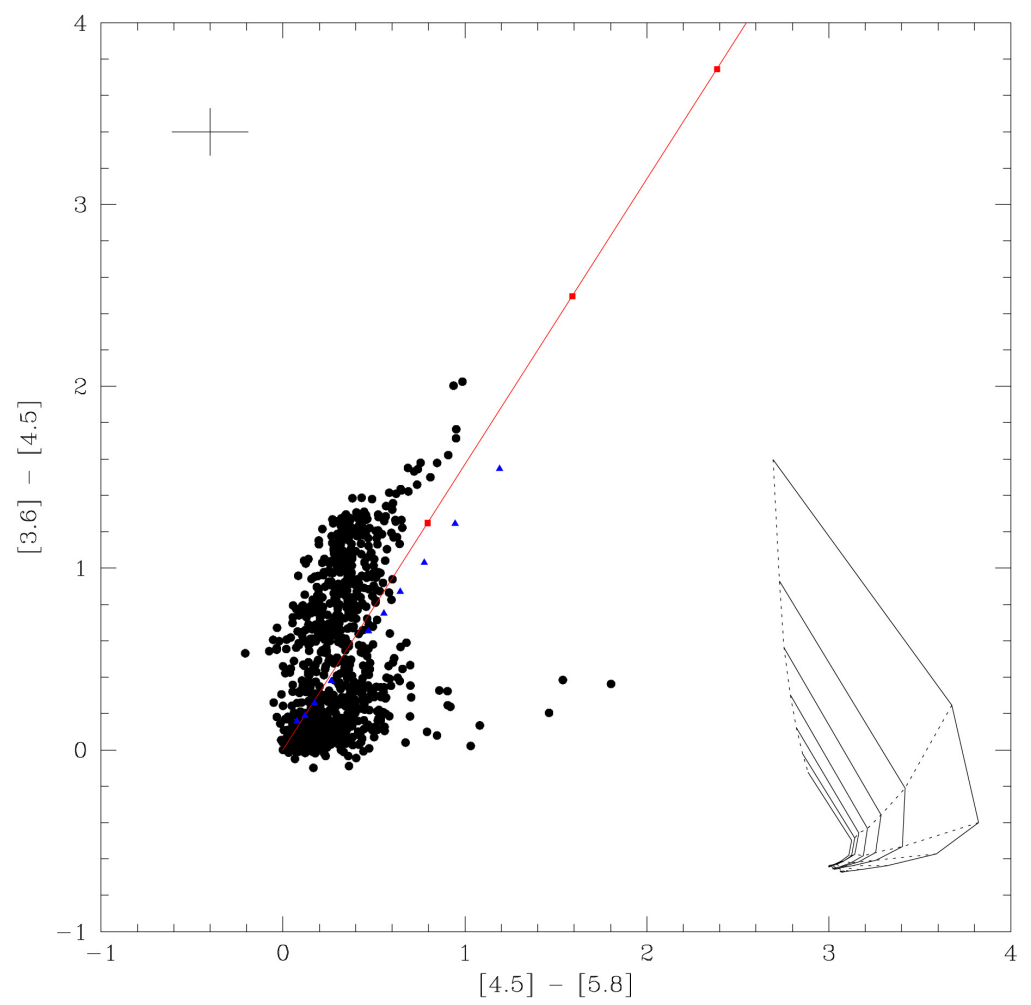

Figure 1. Infrared color of reflection nebula L1527 (filled circles). The nebular colors are measured for every 3.0 " $\times 3.0$ " region. Stellar photospheres are located close to $(0,0)$ for any spectral type. A solid red line from the origin indicates the direction of interstellar extinction. Filled red squares correspond to $A_{\mathrm{V}}=100,200$, and 300 mag, respectively. Filled blue triangles show colors of blackbodies with temperatures between 1000 and $500 \mathrm{~K}$. The $\mathrm{PAH}$ colors predicted by the model are also shown at bottom right. The color of L1527 is consistent with the colors of Class I and Class II sources.

diagram distinguishes between a reflection nebula and an emission nebula containing PAH features.

From the figure, the amount of extinction is estimated. The photospheric color of the protostar IRAS $04368+2557$ is assumed to be $(0,0)$, the same color as dwarfs and giants. We consider that the distance from the points of the nebular color to the line perpendicular to the extinction vector and through the origin corresponds to the extinction in the path from the central star through the nebula to us. Figure 2 shows the extinction map of the nebula L1527. The outflows sweep up the material in the envelope of the protostar, forming a cavity. The wall of the cavity or the residuals in the cavity reflects the light from the central star. Near the central star, the extinction is as large as 150 mag in the $V$ band. The extinction of the eastern outflow is slightly less than that of the western outflow. This estimate is consistent with a near-infrared image showing that the eastern outflow is brighter than the western outflow [10].

\subsection{W5-East}

W5 is an intensively investigated HII region. It is located at a distance of $2.2 \mathrm{kpc}$ in the Perseus arm and constitutes a chain of molecular clouds with W3 and W4. 


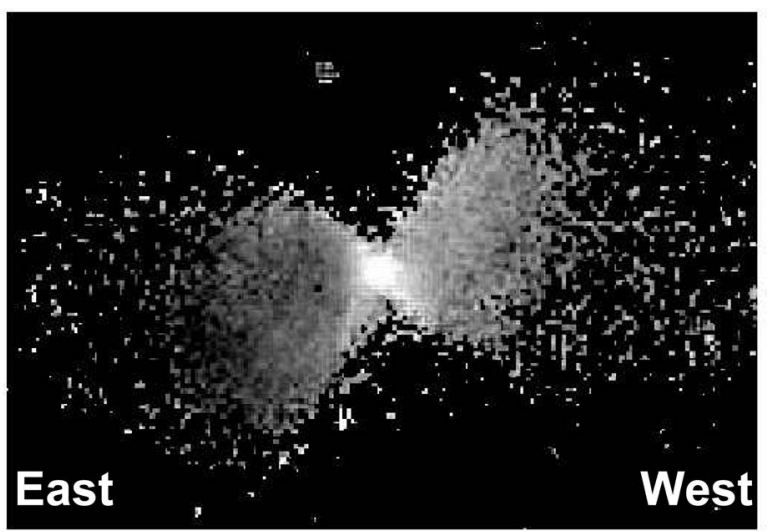

Figure 2. Extinction map of protostar in L1527. The field of view is 179 " $\times 123$ ". A protostar is located at the center of the image. $A_{\mathrm{V}}=20$ mag and $A_{\mathrm{V}}=150 \mathrm{mag}$ are shown in black and white, respectively. Near the protostar, the extinction is as large as 150 mag in the $V$ band. Outflows are located to the east (left) and west (right) of the central star and sweep up the material in the envelope of the protostar. The extinction of the eastern outflow is slightly less than that of the western outflow. In near-infrared wavelengths, the eastern outflow is brighter than the western outflow.

[11] searched for point sources in this region using Spitzer IRAC and MIPS images. They identified 2064 young stellar objects on the basis of their infrared spectral energy distributions. The majority of young stellar objects with infrared excess belong to clusters with more than 10 members.

W5 consists of two circular H II regions, W5-East (W5-E) and W5-West. The ionizing star in W5-E is an $\mathrm{O} 7 \mathrm{~V}$ star, $\mathrm{BD}+59^{\circ} 0578$. We investigate the diffuse emission of the nebulae in the northeast region of W5-E. In this region, two bright rim clouds (BRCs) have been identified [12]. BRC 13 is classified as a Type B cloud, and BRC 14 is identified as a Type A cloud according to their morphology. It is believed that a BRC evolves from Type A through Type B and then Type C [13]. A cluster of massive young stars, AFGL 4029, is located in BRC 14. [14] revealed sequential formation of low-mass stars in this cluster. Figure 3 is a pseudo-color map of the northeast region of W5-E. Nebulae facing the HII region are bright in the IRAC 3 bands. We selected several small regions to investigate the nebular color (Figure 4). Figure 5 shows a color-color diagram of the selected regions of W5-E. The colors of the nebulae are not consistent with the color of the photosphere with extinction or with blackbody radiation. The [4.5] - [5.8] colors of the nebulae are redder than the colors of the photosphere and a blackbody.

DL07 calculated the IRAC colors of interstellar dust irradiated by starlight. They considered a mixture of amorphous silicate and graphitic dust. The size distribution of the silicate and graphitic dust follows the power law distributions presented by [15]. In this distribution, the volume ratio of silicate dust to graphite dust is 1.44. In addition, DL07 included two populations of small carbonaceous dust; both follow lognormal distributions with peaks at 4.0 and $20 \AA$. They constructed seven models of the Milky Way with various mass fraction of small dust $(0.47 \%<q<4.58 \%)$. Small carbonaceous dust has PAH-like properties. 


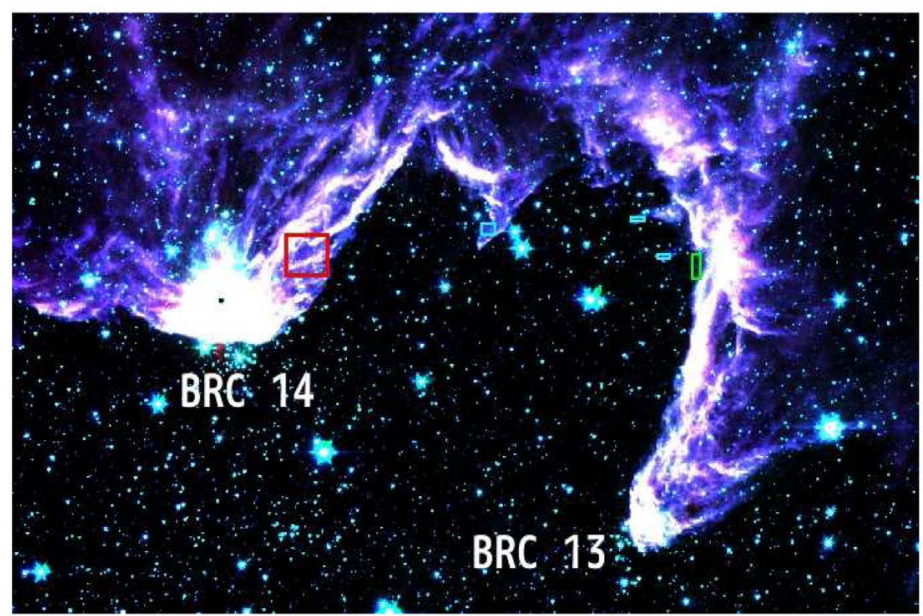

Figure 3. Pseudo-color image of northeast region of W5-E constructed from IRAC 3.6, 4.5 , and $5.8 \mu \mathrm{m}$ images. The field of view is $22.1^{\prime} \times 14.3^{\prime}$. The regions whose colors are discussed on the color-color diagram are indicated by boxes (red: BRC 14, green: bay-like region, blue: $\mathrm{B}$ - and C-like BRCs). The exciting star is located outside the frame below the bottom of the figure.

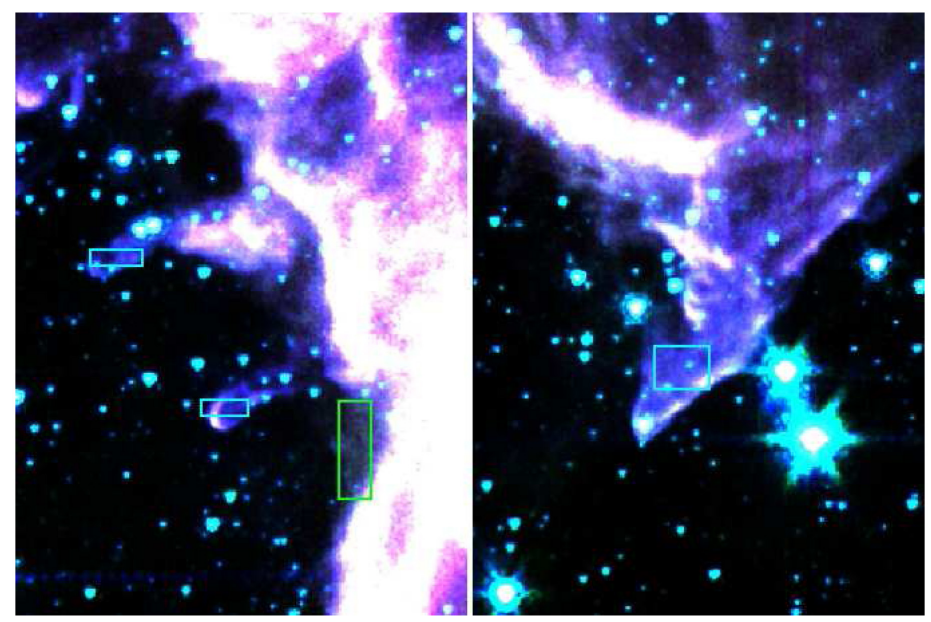

Figure 4. Close-up view of the marked regions in Figure 3. The field of view of both images is $2.7^{\prime} \times 3.6^{\prime}$. The bay-like region is indicated by a green box, and B- and C-like BRCs are indicated by blue boxes.

Heating of dust by starlight is considered. Its strength is parameterized by the factors of the interstellar radiation field ( $\left.U_{\text {ISRF }},[16]\right)$. They presented infrared spectra of dust with various mass fractions of small dust and starlight intensities. The vibration modes of $\mathrm{PAH}$ produce strong emission features at 3.3, 6.2, 7.6, 8.6, and $11.3 \mu \mathrm{m}$. The intensities of these features change with the temperature of the dust. A small dust grain is easily heated by absorption of a few UV photons or a single UV photon and then emits strong PAH features at short wavelengths. Thus, the ratio of the PAH emission feature and the color of specific photometric bands is a function of the fraction of small dust and the intensity of the UV radiation.

The observed colors of the nebulae are consistent with the model colors of 


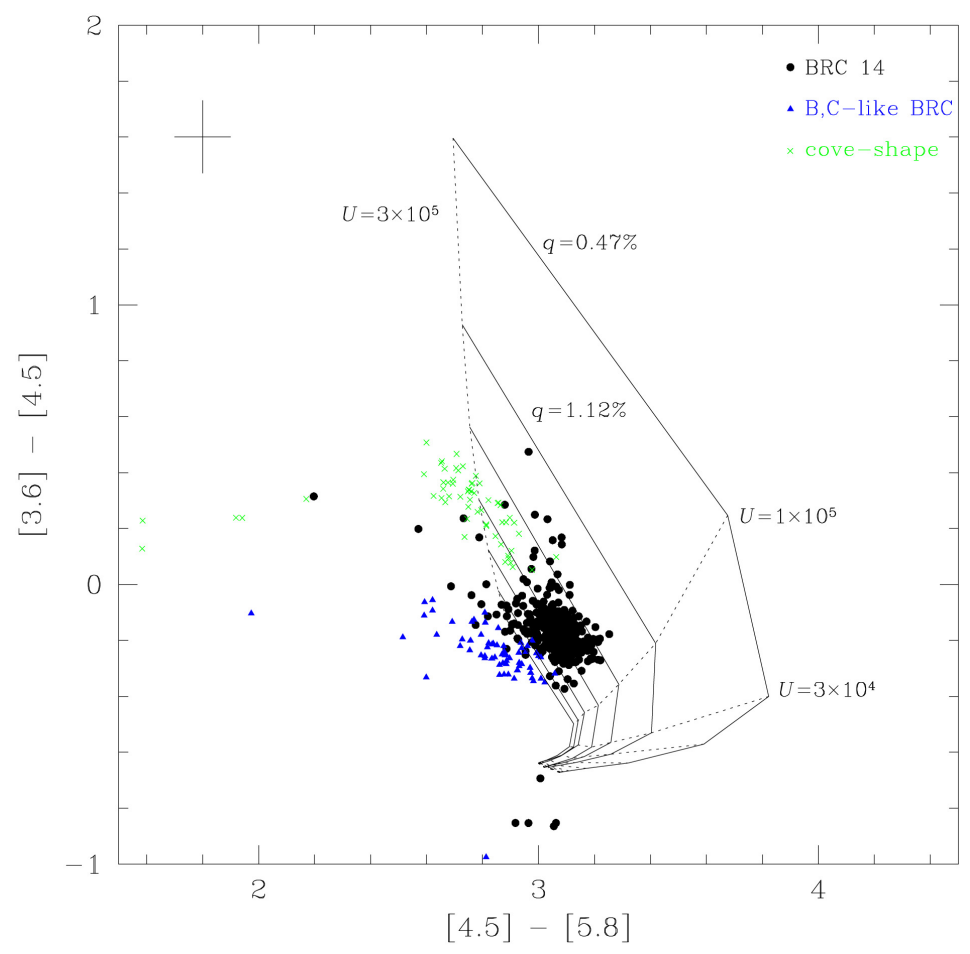

Figure 5. Infrared colors of the marked regions of the nebulae in W5-E. Colors of different nebulae are shown by different symbols. Over plotted are the model color of dust containing PAH. The solid lines show the model colors of various small dust fractions ( $q$ $=0.47 \%, 1.12 \%, 1.77 \%, 2.50 \%, 3.19 \%, 3.90 \%$, and $4.58 \%)$. The nebula containing abundant small dust is bluer. The dotted lines indicate the model colors with various UV radiation levels between $10^{2}$ times and $3 \times 10^{5}$ times the interstellar UV radiation in steps of 0.5 on a logarithmic scale. The nebulae are bright with PAH emission bands.

DL07 (Figure 5). The nebulae are emission nebulae. The colors at different positions in the nebulae are located at different loci in the diagram. The colors of the bay-like region are located at the top-left position representing a part of BRC 14 in the color-color diagram. This indicates that the small dust fraction is small and the UV radiation is strong in the bay-like region compared to those in BRC 14. The colors of the B- and C-like BRCs are located below and to the left of the color of BRC 14 in the diagram. This indicates that the B- and C-like BRCs have an abundant population of small dust irradiated by or a single UV photon and then emits strong PAH features at short wavelengths. Thus, the ratio of the PAH emission feature and the color of specific photometric bands is a function of the fraction of small dust and the intensity of the UV radiation.

The observed colors of the nebulae are consistent with the model colors of DL07 (Figure 5). The nebulae are emission nebulae. The colors at different positions in the nebulae are located at different loci in the diagram. The colors of the bay-like region are located at the top-left position representing a part of BRC 14 in the color-color diagram. This indicates that the small dust fraction is small and the UV radiation is strong in the bay-like region compared to those in BRC 14. The colors of the B- and C-like BRCs are located below and to the left of the color of BRC 14 in the diagram. This indicates that the B- and C-like BRCs have 
an abundant population of small dust irradiated by strong UV radiation.

The strength of the UV radiation is estimated from the nebular color. We calculated the mean color of $5 \times 5$ pixel (3.0" $\times 3.0$ "). The observed colors of the nebulae are compared with the model color of DL07. The model-calculated PAH fluxes for single UV strengths are up to $3 \times 10^{5}$ times the interstellar value. We excluded a pixel if its observed color did not match the model color of DL07. We also ignored pixels near the bright stars, which are masked in the data reduction procedure. Figure 6 shows the spatial distribution of the UV strength. As expected, the edge of the nebulae facing the HII region show colors indicating strong UV radiation. At the inner part of the nebulae, the colors are consistent with the model color with weak UV radiation. Most of the UV photons from the exciting star are thought to be absorbed and scattered by dust in the molecular cloud. We also estimate the small dust fraction as described above. Figure 7 shows

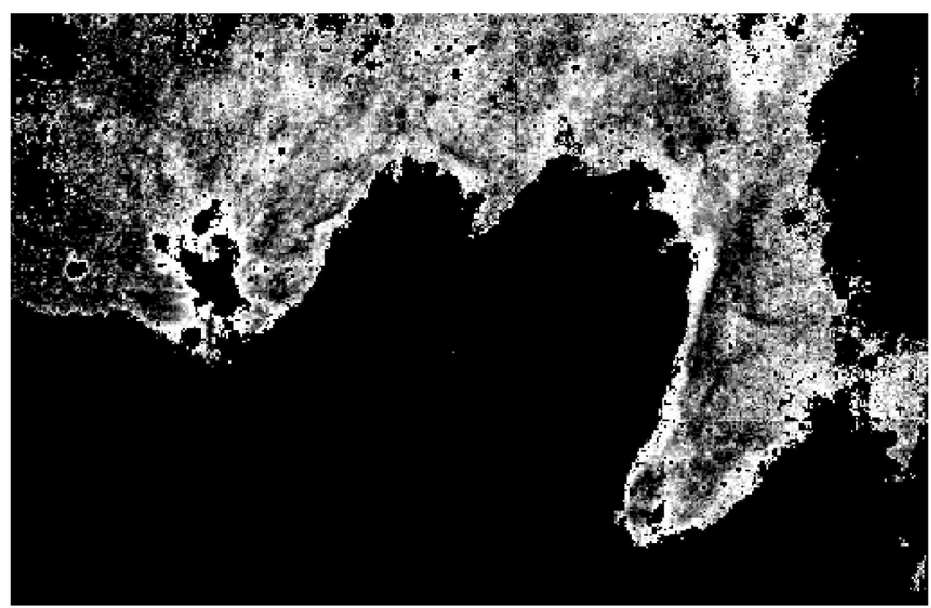

Figure 6. UV irradiation of the nebulae. $\log (U)=5.15 \mathrm{mag}$ and $\log (U)=5.30 \mathrm{mag}$ are depicted in black and white, respectively. The edge of the nebulae facing the HII region are irradiated by strong UV radiation.

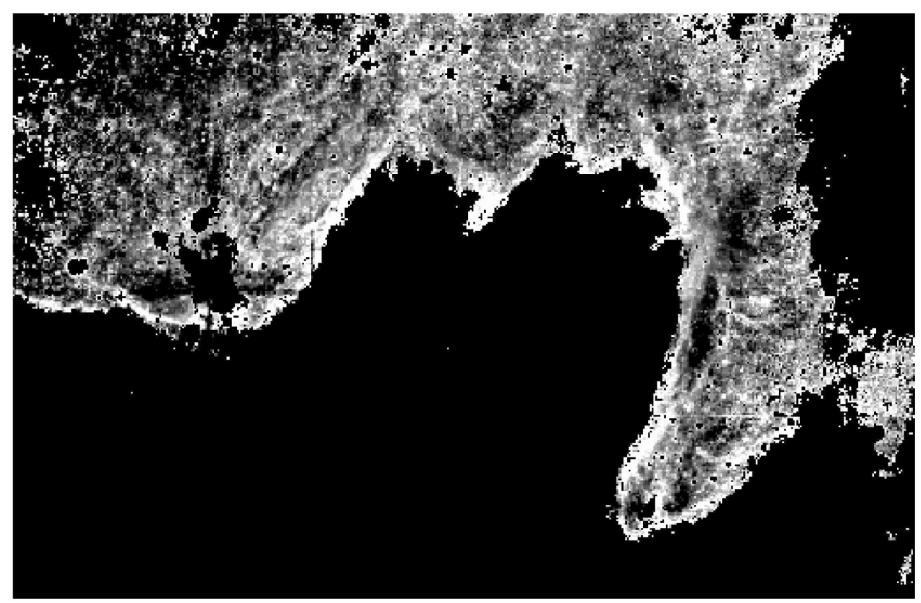

Figure 7. Spatial distribution of small dust fraction. $q=1.5 \%$ mag and $q=3.0 \%$ mag are depicted in black and white, respectively. The map indicates a large small dust fraction at the edge of the nebulae. 
the spatial distribution of the small dust fraction in W5-E. The distribution is not uniform in the nebulae. At the inner part of the nebulae, the small dust fraction is small, and at the edge of the nebulae, it is large. This inhomogeneity is especially obvious for BRC 13, a Type B BRC.

The fact that small dust is more abundant at the edge of the nebulae than in the inner part of the nebulae indicates that large dust grains at the surface of the molecular clouds are reduced to small dust grains by UV radiation from the early-type star.

\section{Discussion}

Our claim that dust grains at the surface of the molecular cloud are destroyed by UV radiation emanating from an early-type star is deduced from the nebular color in the flux-calibrated images. To estimate the precise flux of the nebulae, we subtracted the flux of the HII region adjacent to the nebulae. We examined whether the uncertainty in the flux of the HII region challenges our claim. We added or subtracted a flux representing 5 times the standard deviation of the flux of the HII region and then estimated the strength of the UV radiation and fraction of small dust. Even after we added or subtracted the flux, the claim that small dust is abundant at the surface of the molecular cloud remained valid. Diffuse emission appears even in the HII region (see Figure 1 of [11]). Even if the UV strength and dust population are estimated using the original PBCD images, the spatial distribution of the dust population does not change; i.e., small dust is abundant at the surface of the molecular cloud.

The overabundance of small dust at the surface of the molecular cloud is deduced from the "blue" infrared colors at the edge of the nebula. Nebulae have different infrared colors not only with different small dust fractions and different $\mathrm{UV}$ radiation strengths but also with different amounts of extinction. The blue color at the edge of the nebula is naturally thought to be produced by the low extinction. At the edge of the nebulae, the small dust fraction is as high as $4 \%$, whereas at the inner part of the nebulae, the fraction is as low as $2 \%$ and reaches $1.5 \%$ locally. The [4.5] - [5.8] color difference between the edge and the region with $q=1.5 \%$ is about $0.2 \mathrm{mag}$ (Figure 5). These observations can be reproduced if the inner part of the nebulae has an extinction of 30 mag in the $V$ band. [14] conducted a near-infrared imaging survey of the AFGL 4029 cluster in BRC 14. They detected 605 sources, most of which are classified as background stars. The visual extinction of a star can be estimated from the color difference between the observed color and the intrinsic color of a dwarf or a giant. The color-color diagram of the sources detected in [14] indicated that the largest visual extinction is $\sim 20 \mathrm{mag}$. The average extinction of the sources in the AFGL 4029 cluster and between the cluster and the bright rim is 7.8 mag in the $V$ band. Thus, the visual extinction does not produce the infrared color difference between the edge and the inner part of the nebula. Note that our line of sight and the incident direction of UV radiation from the early-type star to the molecular cloud are different. Nevertheless, there is no robust evidence that the observed 
color difference of the infrared nebula is totally reproduced by the difference in the extinction. We think that the observed color difference is caused, at least partially, by the spatial inhomogeneity of the small dust fraction.

The process of dust destruction has been intensively investigated. [17] claimed that thermal evaporation of dust due to strong radiation from an exciting star is likely to be unimportant even in an HII region. Similar arguments were given in [18]. On the other hand, recent observational studies indicated spatial variation of the physical or chemical state of PAH. [19] observed the M17-Southwest photodissociation region with the Infrared Space Observatory Short Wavelength Spectrometer. They found that all the PAH features peak at the boundary of the HII region and the molecular cloud, indicating spatial variation of the PAH abundance. From the flux ratios of the PAH features, they concluded that most of the PAHs are ionized and almost fully hydrogenated in the HII region and at the boundary. [20] constructed a radiative transfer model of ionized hydrogen regions and fitted it to the infrared spectra of the HII region RCW 120. From the spatial variation of the PAH emission features, they concluded that PAHs are destroyed in the ionized region on a timescale of $30 \mathrm{Myr}$. Observational study of spatially resolved dust size distributions in molecular clouds under different UV environments will conclusively identify the dust destruction process.

\section{Conclusions}

We investigated the infrared colors of nebulae associated with star-forming regions using Spitzer IRAC images.

- The color of the diffuse emission of L1527 is consistent with a photospheric color with large extinction. The central source of the emission is a low-mass protostar, which does not emit strong UV radiation that excites PAHs.

- The nebulae in W5-E facing the HII region are bright in the infrared. Their color is not uniform. A comparison with the model color of small dust emission revealed that the nebulae are bright in PAH emission features. The nebular color indicated that the nebulae facing the HII region are irradiated by strong UV radiation and contain a large population of small dust. We claim that the dust grains at the surface of the molecular cloud are destroyed by UV radiation from an early-type star.

\section{Acknowledgements}

We thank the editor and the referee for their comments. This work is based on observations made with the Spitzer Space Telescope, which was operated by the Jet Propulsion Laboratory, California Institute of Technology under a contract with NASA. This work was supported by JSPS KAKENHI Grant Number JP24540231.

\section{Conflicts of Interest}

The authors declare no conflicts of interest regarding the publication of this paper. 


\section{References}

[1] Mathis, J.S., Rumpl, W. and Nordsieck, K.H. (1977) The Size Distribution of Interstellar Grains. Astrophysical Journal, 217, 425-433. https://doi.org/10.1086/155591

[2] Nakajima, Y., et al. (2003) Deep Imaging Observations of the Lupus 3 Cloud: Dark Cloud Revealed as Infrared Reflection Nebula. Astrophysical Journal, 125, 1407-1417. https://doi.org/10.1086/367913

[3] Tanii, R., et al. (2012) High-Resolution Near-Infrared Polarimetry of a Circumstellar Disk around UX Tau A. Publ. of Astronomical Society of Japan, 64, Article ID: 124. https://doi.org/10.1093/pasj/64.6.124

[4] Boersma, C., Bregman, J.D. and Allamandola, L.J. (2013) Properties of Polycyclic Aromatic Hydrocarbons in the Northwest Photon Dominated Region of NGC 7023. I. PAH Size, Charge, Composition, and Structure Distribution. Astrophysical Journal, 769, Article ID: 117. https://doi.org/10.1088/0004-637X/769/2/117

[5] Draine, B.T. and Li, A. (2007) Infrared Emission from Interstellar Dust. IV. The Silicate-Graphite-PAH Model in the Post-Spitzer Era. Astrophysical Journal, 657, 810-837. https://doi.org/10.1086/511055

[6] Draine, B.T., et al. (2007) Dust Masses, PAH Abundances, and Starlight Intensities in the SINGS Galaxy Sample. Astrophysical Journal, 663, 866-894.

https://doi.org/10.1086/518306

[7] Megeath, S.T. et al. (2004) Initial Results from the Spitzer Young Stellar Cluster Survey. Astrophysical Journal, 154, 367-373. https://doi.org/10.1086/422823

[8] Chapman, N.L. and Mundy, L.G. (2009) Deep JHKs and Spitzer Imaging of Four Isolated Molecular Cloud Cores. Astrophysical Journal, 699, 1866-1882. https://doi.org/10.1088/0004-637X/699/2/1866

[9] Hartmann, L., et al. (2005) IRAC Observations of Taurus Pre-Main-Sequence Stars. Astrophysical Journal, 629, 881-896. https://doi.org/10.1086/431472

[10] Tamura, M., et al. (1996) Interferometric Observations of Outflows from Low-Mass Protostars in Taurus. Astrophysical Journal, 112. 2076-2085. https://doi.org/10.1086/118164

[11] Koenig, X.P., et al. (2008) Clustered and Triggered Star Formation in W5: Observations with Spitzer. Astrophysical Journal, 688, 1142-1158. https://doi.org/10.1086/592322

[12] Sugitani, K., et al. (1989) Star formation in Bright-Rimmed Globules-Evidence for Radiation-Driven Implosion. Astrophysical Journal, 342, L87-L90. https://doi.org/10.1007/BFb0119459

[13] Miao, J., et al. (2009) An Investigation on the Morphological Evolution of Bright-Rimmed Clouds. Astrophysical Journal, 692, 382-401. https://doi.org/10.1088/0004-637X/692/1/382

[14] Matsuyanagi, I., et al. (2006) Sequential Formation of Low-Mass Stars in the BRC 14 Region. Publications of the Astronomical Society of Japan, 58, L29-L34. https://doi.org/10.1093/pasj/58.4.L29

[15] Weingartner, J.C. and Draine, B.T. (2001) Dust Grain-Size Distributions and Extinction in the Milky Way, Large Magellanic Cloud, and Small Magellanic Cloud. Astrophysical Journal, 548, 296-309. https://doi.org/10.1086/318651

[16] Mathis, J.S., Mezger, P.G. and Panagia, N. (1983) Interstellar Radiation Field and Dust Temperatures in the Diffuse Interstellar Matter and in Giant Molecular Clouds. Astronomy \& Astrophysics, 128, 212-229. 
[17] Salpeter, E.E. (1977) Formation and Destruction of Dust Grains. Annual Review of Astronomy \& Astrophysics, 15, 267-293.

https://doi.org/10.1146/annurev.aa.15.090177.001411

[18] Draine, B.T. and Salpeter, E.E. (1979) Destruction Mechanisms for Interstellar Dust. The Astrophysical Journal, 231, 438-455.

[19] Verstraete, L., et al. (1996) SWS Spectroscopy of Small Grain Features across the M17-Southwest Photo Dissociation Front. Astronomy \& Astrophysics, 315, L337-L340.

[20] Pavlyuchenkov, Y.N., Kirsanova, M.S. and Wiebe, D.S. (2013) Infrared Emission and the Destruction of Dust in HII Regions. Astronomy Reports, 57, 573-585.

https://doi.org/10.1134/S1063772913070056 